\title{
Bone Mineral Density at the Distal Femur and Proximal Tibia and Related Factors During the First Year of Spinal Cord Injury
}

This article was published in the following Dove Press journal: International Journal of General Medicine

\author{
Xin Zheng $\mathbb{D}^{1,2}$ \\ Yanyan $\mathrm{Qi}^{1,2}$ \\ Hongjun Zhou ${ }^{2,3}$ \\ Haiqiong Kang ${ }^{2,3}$ \\ Yanming Tong ${ }^{1,2}$ \\ Lina $\mathrm{Bi}^{1,2}$
}

'Department of Endocrinology, China Rehabilitation Research Center, Beijing, People's Republic of China; ${ }^{2}$ School of Rehabilitation Medicine, Capital Medical University, Beijing, People's Republic of China; ${ }^{3}$ Department of Spinal Cord Injury Rehabilitation, China Rehabilitation Research Center, Beijing, People's Republic of China
Correspondence: Xin Zheng

Department of Endocrinology, China Rehabilitation Research Center, No. 10 Jiaomen North Road, Fengtai District, Beijing, 100068, People's Republic of China

Tel +86-I38II568977

Email zhengxincrrc@।26.com

Hongjun Zhou

Department of Spinal Cord Injury Rehabilitation, China Rehabilitation

Research Center, No. 10 Jiaomen North

Road, Fengtai District, Beijing, I00068,

People's Republic of China

Tel +86-0 I0-87563 I I5

Email zhjcrrc@I63.com
Background: Spinal cord injury (SCI) can lead to disuse osteoporosis. The most vulnerable sites for fragility-induced fractures are the distal femur (DF) and proximal tibia (PT). The aim of this study was to evaluate changes in bone mineral density (BMD) at the DF and PT, as well as related factors, during the first year of SCI.

Patients and Methods: Thirty-six SCI patients within 12 months of their injury were selected, as were 36 healthy controls. The dual-energy X-ray absorptiometry was used to measure BMDs at the DF, PT, and hip of all subjects. According to the duration of SCI when receiving DXA scan, 36 SCI patients were divided into three subgroups. The BMDs of overall patients and subgroups were compared to those of controls. Biochemical markers of bone metabolism were detected in SCI patients.

Results: The BMDs at the DF, PT, and hips of overall SCI patients were significantly lower than those of controls. The percentage difference of BMD between SCI patients and controls at the DF and PT was higher than at the hip. The BMD at the PT of SCI within 6 weeks postinjury was lower than that of controls. The BMDs at the DF and PT of SCI during 6 weeks-3 months post-injury were lower than those of controls. Whereas there was no difference in the $\mathrm{BMD}$ at the hip during the first 3 months of SCI. Age and 25OHD were the influencing factors of DF BMD. Age and gender were found to influence PT BMD.

Conclusion: The rapid loss of BMD at the PT and DF during the first year of SCI occurred significantly earlier than that of the hip. It is recommended to monitor the BMD of DF and PT in early-stage SCI patients, combined with detection of biochemical markers of bone metabolism.

Keywords: spinal cord injury, bone mineral density, distal femur, proximal tibia, disuse osteoporosis

\section{Introduction}

Spinal cord injury (SCI) is a serious disabling disease. Disuse osteoporosis after SCI leads to an increased risk of fracture. Previous studies have reported that $8.7 \%-$ $50 \%$ of SCI patients have fractures. ${ }^{1-3}$ The distal femur (DF) and proximal tibia (PT) are the most vulnerable sites for fractures in SCI patients. Patients with SCI usually sit in wheelchairs, and DF and PT have the highest risk of low-impact fragility fractures. ${ }^{4}$ The clinical detection of bone mineral density (BMD) in patients with SCI mostly focuses on the traditional proximal femur and lumbar spine. Previous studies have found that at 8-24 weeks after SCI, there is no change in BMD of the proximal femur between SCI patients and healthy controls, while 
lumbar-spine BMD is also often not decreased. ${ }^{5-7}$ Thus, only detecting BMD of the proximal femur cannot clearly show evidence of bone demineralization in the early stage of SCI. As such, it is of great clinical significance to determine changes in BMDs at the PT and DF in SCI patients and to detect bone loss in areas with high fracture risk as soon as possible.

There have been no previous studies that have reported BMDs at the DF and PT in Chinese patients with SCI. Thus, changes in BMDs at the DF and PT, as well as related factors, and whether the BMDs at the DF and PT decrease at the same rate as that of the proximal femur in Chinese patients with SCI remain unclear. Previous studies have shown that most decline in BMD is observed in the early stage of SCI, especially within one year after injury. ${ }^{8,9}$ Hence, this period has become an important treatment window for osteoporosis intervention. ${ }^{10}$ The aim of the present study was to analyze changes in BMDs at the DF and PT, as well as related factors, in Chinese patients with SCI during the first year, and to provide clinical guidance for the early prevention and treatment of SCI-induced disuse osteoporosis.

\section{Patients and Methods}

\section{Patients}

A total of 36 SCI patients within 12 months post-injury hospitalized in China Rehabilitation Research Center from April 2018 to August 2019 were selected for our present study. This sample included 26 males and 10 females, with an average age of $(36.78 \pm 10.54)$ years and an average bodymass index (BMI) of $(23.02 \pm 3.76) \mathrm{kg} / \mathrm{m}^{2}$. According to the American Spinal Injury Association (ASIA) injury scale, ${ }^{11}$ 20 patients displayed a complete lesion (grade A) and 16 patients displayed an incomplete lesion (8 B, 5 C, and 3D). All patients underwent similar, routine rehabilitation training during hospitalization, including physiotherapy and occupational therapy. Exclusion criteria were as follows: any metabolic bone disease (eg, thyroid disease, parathyroid disease, hypercortisolism, osteomalacia, or Paget disease), history of pathological fracture, diabetes, autoimmune disease, severe liver or kidney disease, any malignant tumor, use of drugs affecting bone metabolism (eg, glucocorticoids, immunosuppressant, antiepileptics, anticoagulants, and antiosteoporosis medications), or postmenopausal status.

This is mainly a cross-sectional study. The BMD of 36 SCI patients was measured and compared with that of the controls. The SCI patients underwent a DXA scan at different timepoints. According to the duration of SCI when receiving DXA scan, the 36 patients were further divided into three subgroups: within 6 weeks post-injury $(\mathrm{n}=15), 6$ weeks to 3 months post-injury $(\mathrm{n}=10)$, and $3-12$ months post-injury $(\mathrm{n}=11)$. The BMD of the three subgroups was compared to that of the controls. In addition, 8 of the 36 patients completed two DXA scans, and the interval between the two scans was 6 months. Longitudinal observations of BMD were performed in these eight subjects.

The control participants were recruited from among the healthy, voluntary hospital staff. Thirty-six healthy controls were selected by age, gender, and BMI to match the SCI group. The average age of the control group was $(36.11 \pm 10.63)$ years, and the average BMI was (23.27 $\pm 2.84) \mathrm{kg} / \mathrm{m}^{2}$. There was no statistical difference in age, sex ratio, or BMI between the SCI group and the control group ( $\mathrm{P}>0.05)$. This study was approved by the Ethics Committee of China Rehabilitation Research Center. The study conformed to the ethical guidelines of the Declaration of Helsinki. All subjects provided written informed consent before participating.

\section{Medical History and Clinical Data Collection}

The medical history and collected clinical data of the patients included gender, age, postinjury duration, cause of injury, injury severity, type of paralysis, comorbid diseases, height $(\mathrm{H})$, weight $(\mathrm{W}), \mathrm{BMI}$, and $\mathrm{BMI}=\mathrm{W} / \mathrm{H}^{2}\left(\mathrm{~kg} / \mathrm{m}^{2}\right)$.

\section{Laboratory Examinations}

Blood samples were collected from SCI patients on the same day as their DXA scan. After fasting overnight, each SCI patient provided a venous blood sample. Biochemical markers of bone metabolism were analyzed from the blood serum. An automatic biochemical analyzer (BS-2000M, Mindray, China) was used to detect serum calcium (Ca), phosphorus (P), and alkaline phosphatase (ALP) in patients with SCI. An electrochemiluminescence immunoassay analyzer (Cobas E411, Roche, Switzerland) was used to detect 25hydroxy vitamin D (25-OHD), osteocalcin (OC), N-terminal propeptide of type-I procollagen (PINP), collagen type-I C-terminal telopeptide (CTX), and parathyroid hormone (PTH).

\section{Assessment of BMDs}

The BMDs of SCI patients and control subjects were measured by dual-energy X-ray absorptiometry (DXA) 
using a DXA scanner (Discovery-Wi, Hologic, USA). The subjects were placed in a supine position, and the BMDs at the DF, PT, and hip (including those of the femoral neck and total hip) were measured. The lower limbs were stabilized in full extension during the DXA scan of DF and PT. The hip standard module automatically detected the region of interest (ROI) of the hip, which is routinely performed during clinical examinations of osteoporosis. The DF- and PT-imaging areas were considered to be the area of the distal third of the femur and the proximal third of the tibia, respectively. ${ }^{4}$ At present, there is no standard module or measurement method for determining BMDs of these regions, and there is no unified and recognized standardized method to obtain ROIs from these regions based on DXA. Usually, severe bone loss mainly occurs in the epiphysis of the weight-bearing bone, and the most likely fractured area is also the epiphysis. ${ }^{12}$ Thus, the epiphyses of the DF and PT were selected in the present study. For the PT, the distal edge of the ROI was the tibiafibular intersection and the proximal edge was the tibial plateau. For the DF, the distal edge of the ROI was the upper edge of the patella (to avoid the influence of the patella on the BMD at DF), and the proximal edge was set by $30 \%$ of the height of the ROI in the PT. A metalremoval protocol was used to capture the DF and PT of the right leg as separate imaging areas in a thin-scan mode. Then, we used manual segmentation to separate the target bone from the tissue, air, and other bones in the scanning field of view. DXA was ordered systematically and calibrated precisely. A quality-assurance phantom supplied by the manufacturer was measured every day to confirm accuracy of the densitometer. Before the start of the experiment, the phantom was scanned 10 times, and the longitudinal fluctuation range of phantom measurement was determined according to the principle of $1.5 \%$ confidence limit (formula: average value of 10 scans of the phantom \pm average value* $1.5 \%$ ). According to this, the value of the phantom was required to be $0.9491-0.9780$. The BMD measurement data of the quality-assurance phantom showed the CV value to be $0.362 \%$. A quality control scan was carried out prior to each set of patient scans. All scanning and analysis were performed by the same operator to ensure consistency and followed standard quality-control procedures.

\section{Statistical Analysis}

All statistical analyses were performed using SPSS 25.0 (SPSS Inc., Chicago, IL, USA). A Shapiro-Wilk test was performed to assess normality of the measurement data. Normally distributed data are expressed as mean \pm standard deviation (SD), whereas non-normally-distributed data are expressed as the interquartile range (IQR). An independent sample $t$-test was used to compare the BMDs between the two groups. A paired $t$-test was used to compare the BMDs of the same patient at different postinjury durations. A Chi-square test was used for gender comparison between two groups. Correlations between the variables were determined via Pearson correlation coefficient or Spearman's rank correlation coefficient tests. Multiple linear regression was used to analyze the influencing factors of BMDs at DF and PT. A $P<0.05$ was considered statistically significant.

\section{Results}

\section{Clinical Characteristics of SCl Patients}

The clinical characteristics of SCI patients are shown in Table 1 . There was one case $(2.78 \%)$ with sufficient $25 \mathrm{OHD}(>30 \mathrm{ng} / \mathrm{mL})$, three cases $(8.33 \%)$ with insufficient $25 \mathrm{OHD}(<30 \mathrm{ng} / \mathrm{mL})$, and 32 cases $(88.89 \%)$ with 25 OHD deficiency $(<20 \mathrm{ng} / \mathrm{mL})$.

Table I Demographic and Clinical Data of SCI Patients

\begin{tabular}{|l|l|}
\hline Cases $(\mathrm{n})$ & 36 \\
Gender (M/F) & $26 / 10$ \\
Age (years) & $37.0 \pm 10.3$ \\
Postinjury duration (months) & $2(0.98,4.45)$ \\
Cause of SCl (traumatic/non-traumatic) & $31 / 5$ \\
Type of paralysis (paraplegia/quadriplegia) & $30 / 6$ \\
Injury severity (complete/incomplete) & $20 / 16$ \\
BMI $\left(\mathrm{kg} / \mathrm{m}^{2}\right)$ & $22.96 \pm 3.67$ \\
DF BMD $\left(\mathrm{g} / \mathrm{cm}^{2}\right)$ & $0.816 \pm 0.155$ \\
PT BMD $\left(\mathrm{g} / \mathrm{cm}^{2}\right)$ & $0.800 \pm 0.159$ \\
Femoral neck BMD $\left(\mathrm{g} / \mathrm{cm}^{2}\right)$ & $0.752 \pm 0.110$ \\
Total hip BMD $\left(\mathrm{g} / \mathrm{cm}^{2}\right)$ & $0.904 \pm 0.149$ \\
Ca $(\mathrm{mmol} / \mathrm{L})$ & $2.34 \pm 0.12$ \\
P (mmol/L) & $1.41 \pm 0.20$ \\
ALP $(\mathrm{IU} / \mathrm{L})$ & $81.10(62.48,125.78)$ \\
$25 O H D(\mathrm{ng} / \mathrm{mL})$ & $14.28 \pm 6.18$ \\
Number/Percentage of $25 \mathrm{OHD}$ deficiency & $32 / 88.89 \%$ \\
CTX (ng/mL) & $1.056 \pm 0.357$ \\
PINP (ng/mL) & $106.10(85.58,145.75)$ \\
OC (ng/mL) & $19.38 \pm 6.88$ \\
PTH (ng/mL) & $20.79(16.85,26.63)$ \\
\hline
\end{tabular}

Abbreviations: $\mathrm{SCl}$, spinal cord injury; $\mathrm{BMI}$, body mass index; DF, distal femur; PT, proximal tibia; BMD, bone mineral density; ALP, alkaline phosphatase; 25OHD, 25-hydroxyvitamin D; CTX, collagen type-I C-terminal telopeptide; PINP, $\mathrm{N}$-terminal propeptide of type-I procollagen; OC, osteocalcin; PTH, parathyroid hormone. 
Comparison of BMDs at the DF, PT, and Hip, Including Femoral Neck and Total Hip, Between SCl Patients and Healthy

\section{Controls}

The BMDs at the PT, DF, and hips of the overall SCI patients were all lower than those of the control group (Figure 1A). Percentage difference from control group in BMDs at the DF $(-12.35 \%)$ and PT $(-14.53 \%)$ was higher than those at the femoral neck $(-8.85 \%)$ and total hip $(-11.80 \%)$. Subgroup analysis showed that the BMD at the PT of SCI patients within 6 weeks post-injury was lower than that of the control group, while there was no difference in the BMD at the DF or hip between the two groups (Figure 1B). The BMDs at the PT and DF of SCI patients during 6 weeks-3 months post-injury were lower than those of the control group, but there was no difference in the BMD at the hip (Figure 1C). The BMDs at the DF, PT, and hip of SCI patients during 3-12 months postinjury were all lower than those of the control group (Figure 1D). The Z-score of femoral neck and total hip was shown in Table 2. The Z-score of femoral neck and total hip gradually decreased with the extension of SCI duration.

\section{Longitudinal Comparison of BMDs at the DF, PT, and Hip in Eight SCI Patients}

Eight patients with SCI underwent DXA testing for the first time within three months after SCI, and DXA testing was performed again six months later. The BMDs at the DF and PT after six months were decreased significantly by $11.4 \%$ and $10.8 \%$, respectively, compared with those of baseline values. The BMDs at the femoral neck and total
A

A All SCl within 12 months $(n=36)$

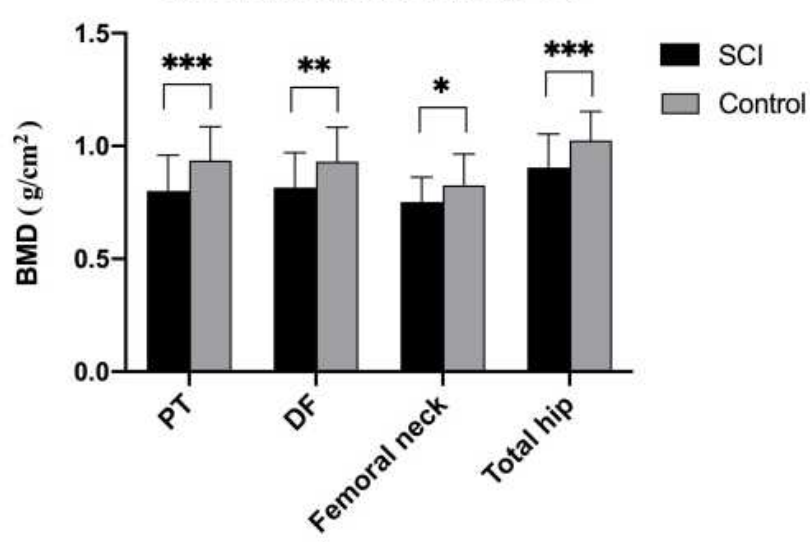

C

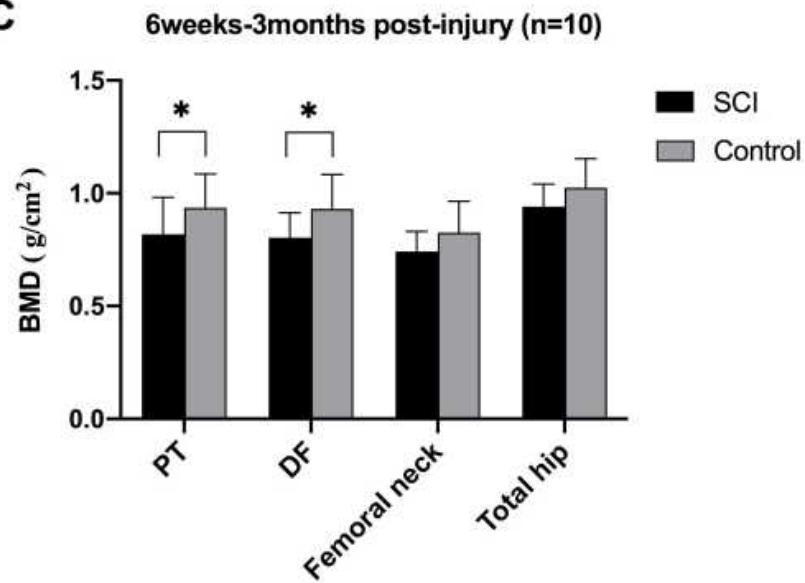

B SCI within 6weeks $(n=15)$

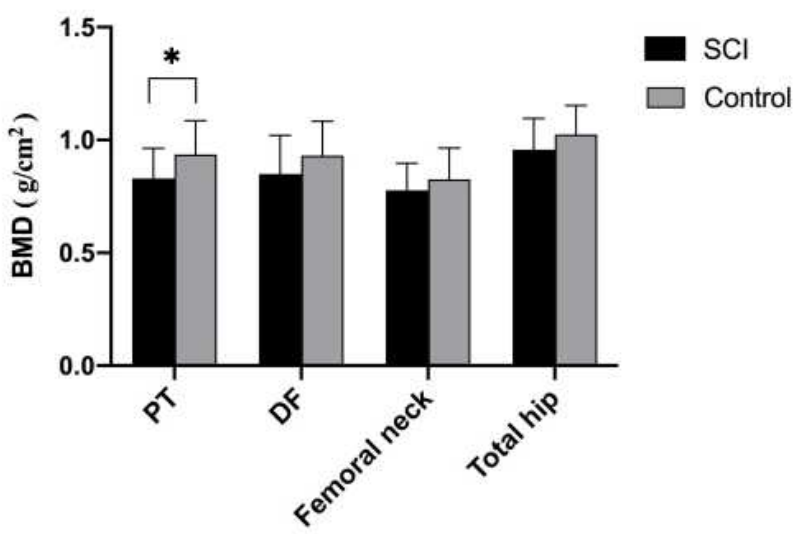

D

3-12months post-injury $(n=11)$

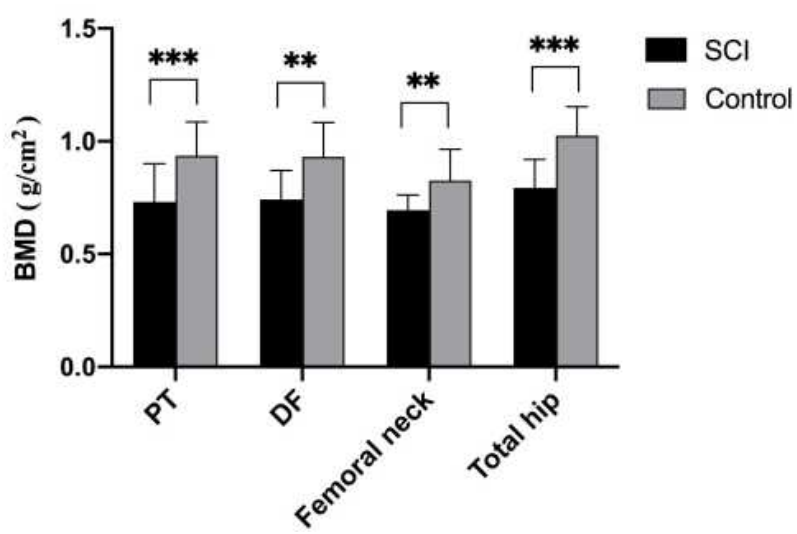

Figure I Comparison of BMDs at the DF, PT, and hip between $\mathrm{SCl}$ patients and healthy controls. (A) Comparison of $\mathrm{BMDs}$ between $\mathrm{All} \mathrm{SCl}$ patients ( $\mathrm{n}=36$ ) and the controls; (B) Comparison of BMDs between SCl patients within 6 weeks post-injury $(n=15)$ and the controls; (C) Comparison of BMDs between SCl patients during 6 weeks-3months post-injury $(n=10)$ and the controls; (D) Comparison of BMDs between $\mathrm{SCl}$ patients during $3-12$ months post-injury $(n=I I)$ and the controls. $* P<0.05$, $* * P<0.01$, $* * * P<0.001$, independent sample $t$-test.

Abbreviations: BMD, bone mineral density; DF, distal femur; PT, proximal tibia; SCl, spinal cord injury. 
Table 2 Z-Score of Femoral Neck and Total Hip in SCl Patients

\begin{tabular}{|l|c|c|c|c|}
\hline & 6 w PI (n=15) & 6 w-3 $\mathbf{~ m ~ P I ~ ( n = 1 0 ) ~}$ & 3-12 $\mathbf{~ m ~ P I ~ ( n = I ~ I ) ~}$ & All SCI (n=36) \\
\hline Z-score of femoral neck & $-0.620 \pm 0.948$ & $-0.660 \pm 0.802$ & $-1.245 \pm 0.474$ & $-0.822 \pm 0.819$ \\
Z-score of total hip & $-0.220 \pm 0.998$ & $-0.330 \pm 0.779$ & $-1.300 \pm 0.867$ & $-0.58 I \pm 1.003$ \\
\hline
\end{tabular}

Abbreviations: SCl, spinal cord injury; Pl, post-injury; $6 \mathrm{w}, 6$ weeks; $6 \mathrm{w}-3 \mathrm{~m}, 6$ weeks to 3 months; 3-12 m, 3-12 months.

hip showed a downward trend of decreases by $3.76 \%$ and $9.13 \%$, respectively, compared with those of baseline values, but these differences were not statistically significant (Table 3). The Z-score of femoral neck $(-1.343$ $\pm 0.493)$ and total hip $(-1.343 \pm 0.887)$ after six months were lower than the Z-score of femoral neck $(-1.171$ $\pm 0.596)$ and total hip $(-0.786 \pm 0.765)$ at baseline.

\section{Correlations Between Clinical Indicators and BMDs at the DF and PT}

The BMD at the DF was negatively correlated with age and PTH and was positively correlated with $25 \mathrm{OHD}$, ALP, and PINP. The $\mathrm{BMD}$ at the PT was negatively correlated with age and was positively correlated with PINP. The BMDs at the DF and PT were each positively correlated with hip BMD (Table 4).

\section{Multiple Linear Regression Analysis of BMDs at the DF and PT}

Multiple linear regression analysis was performed with the $\mathrm{BMD}$ at the DF as the dependent variable, age, gender, PTH, 25OHD, ALP, and PINP as the independent variables, the BMD at the PT as the dependent variables and age, gender, and PINP as the independent variables. The results showed that age and $25 \mathrm{OHD}$ to be the influencing factors of BMD at the DF and age and gender to be the influencing factors of the BMD at the PT (Table 5).

\section{Comparison of BMD Between Complete Lesion and Incomplete Lesion}

There was no significant difference between the complete lesion and incomplete lesion groups (Table 6).

\section{Discussion}

Compared with other common rapid bone-loss conditions, the extremely rapid bone-loss rate after acute SCI is significantly higher than that of postmenopausal osteoporosis, long-term bed rest, and space-flight-induced SCI. ${ }^{13-15}$ The risk of fracture in patients with SCI is twice that of the general population, and the risk of fracture gradually increases with the duration of injury. ${ }^{3,6,16}$ Fractures can lead to hip and knee contractures, osteomyelitis, pressure ulcers, and other complications, further leading to a decline in the mobility of patients with SCI, and adding more difficulties and costs to subsequent rehabilitation. When an accidental collision or a fall from a wheelchair occurs during postural transfer, wheelchair advancement, or rehabilitation training, the DF and PT are the first points of contact with external forces, and therefore are the most common sites for low-energy fragility fractures in daily life. ${ }^{17}$ In clinical evaluation of bone loss after SCI, it is particularly important to locate high-risk areas of fracture and to quantify corresponding rates of bone loss. A previous study found that bone loss was rapid within one year after SCI and reached a new steady-state level within 2-3 years. At this time, compared with that of the normal population, the BMD of SCI patients is decreased by $50 \%$, whereas subsequent bone loss tends to be slow. ${ }^{9,18}$ Therefore, it is important to better understand the characteristics of bone loss during the acute phase of SCI (ie, within one year), as well as to administer early monitoring and interventions during this initial rapid bone-loss period. Analysis of BMDs at the DF and PT of patients with SCI during the first year may be helpful for professionals to better formulate effective early treatment methods to prevent the

Table 3 Longitudinal Comparison of BMDs at the DF, PT, and Hip in Eight SCI Patients

\begin{tabular}{|l|c|c|c|c|}
\hline & DF BMD $\left(\mathbf{g} / \mathbf{c m}^{2}\right)$ & PT BMD $\left(\mathbf{g} / \mathbf{c m}^{2}\right)$ & Femoral Neck BMD $\left(\mathbf{g} / \mathbf{c m}^{2}\right)$ & Total Hip BMD $\left(\mathbf{g} / \mathbf{c m}^{2}\right)$ \\
\hline Baseline & $0.851 \pm 0.114$ & $0.824 \pm 0.128$ & $0.719 \pm 0.083$ & $0.876 \pm 0.126$ \\
After six months & $0.754 \pm 0.147$ & $0.735 \pm 0.178$ & $0.692 \pm 0.077$ & $0.796 \pm 0.136$ \\
Reduction (\%) & 11.40 & 10.80 & 3.76 & 9.13 \\
t & 2.569 & 3.234 & 1.242 & 1.968 \\
P & 0.042 & 0.018 & 0.261 & 0.097 \\
\hline
\end{tabular}

Abbreviations: DF, distal femur; PT, proximal tibia; BMD, bone mineral density. 
Table 4 Correlations Between Clinical Indicators and BMDs at the DF and PT

\begin{tabular}{|l|c|c|c|c|}
\hline \multirow{2}{*}{} & \multicolumn{2}{|c|}{ DF BMD } & \multicolumn{2}{c|}{ PT BMD } \\
\cline { 2 - 5 } & $\mathbf{r}$ & $\mathbf{P}$ & $\mathbf{r}$ & $\mathbf{P}$ \\
\hline Age & -0.457 & 0.005 & -0.503 & 0.002 \\
Time post-injury & -0.24 & 0.158 & 0.236 & 0.167 \\
BMI & 0.025 & 0.884 & 0.238 & 0.162 \\
Ca & 0.256 & 0.150 & 0.091 & 0.616 \\
P & 0.293 & 0.098 & 0.290 & 0.102 \\
ALP & 0.404 & 0.020 & 0.305 & 0.084 \\
25OHD & 0.397 & 0.040 & 0.132 & 0.510 \\
OC & 0.156 & 0.428 & -0.023 & 0.907 \\
CTX & 0.287 & 0.139 & 0.23 & 0.238 \\
PINP & 0.401 & 0.034 & 0.423 & 0.025 \\
PTH & -0.449 & 0.017 & -0.285 & 0.141 \\
Femoral neck BMD & 0.638 & $<0.001$ & 0.706 & $<0.001$ \\
Total hip BMD & 0.547 & 0.001 & 0.631 & $<0.001$ \\
\hline
\end{tabular}

Abbreviations: BMI, body mass index; DF, distal femur; PT, proximal tibia; BMD, bone mineral density; ALP, alkaline phosphatase; 25OHD, 25-hydroxyvitamin D; CTX, collagen type-I C-terminal telopeptide; PINP, N-terminal propeptide of type-I procollagen; OC, osteocalcin; PTH, parathyroid hormone.

occurrence of fractures at high-risk sites and to maintain long-term bone health of SCI patients. DXA is the primary method for clinical assessment of BMD, and it can accurately quantify $\mathrm{BMDs}$ at the $\mathrm{DF}$ and $\mathrm{PT}^{4,5}$

There have been few reports on SCI within one year or during the acute phase, and no relevant reports have been published in regard to the Chinese population in this context. Previous cross-sectional studies on BMDs at the DF and PT have mostly included reports on patients with chronic SCI.
Studies have shown that $30 \%, 70 \%$, and $52 \%$ of bone demineralization were found in the femoral neck, DF, and PT in patients with chronic SCI exceeding one year. ${ }^{8}$ Previous studies have observed a decrease in hip BMD 3-6 months postinjury. ${ }^{5,6}$ This present study showed that the decline in BMDs at the DF and PT occurred earlier than that at the hip. A decrease in the BMD at the PT and DF was observed during the first 3 months of SCI, and the amplitude of decline of BMD at the DF and PT was higher than that at the hip. This indicated that patients with acute SCI experienced rapid and massive loss of bone minerals around the knee, suggesting the importance of early detection of BMD loss at the DF and PT. It may represent a treatment time window during which interventions (including drugs and/or physical therapy) can be performed to control and delay the progression of osteoporosis. The decline of BMD was found to occur earlier at the PT than in other areas, suggesting that Chinese patients with SCI may pay more attention to the changes in BMD at the PT. However, changes in hip BMD were not observed until 3 months post-injury, which was consistent with previous studies, suggesting that only routine hip detection would miss the best time to monitor and intervene for BMD changes in high-risk areas of knee peripheral fractures. Z-score of femoral neck and total hip gradually decreased with the duration of SCI. This suggested that the BMD showed a continuous downward trend during the first year of SCI. Since most fractures in SCI patients usually occur two years or more after injury, early intervention may reduce the patient's future fracture risk. ${ }^{2}$

Table 5 Multiple Linear Regression Analysis of BMDs at the DF and PT

\begin{tabular}{|l|c|c|c|c|c|c|}
\hline & Variable & Regression Coefficient & Standard Error & Standard Regression Coefficient & $\mathbf{t}$ & $\boldsymbol{P}$ \\
\hline \multirow{2}{*}{ DF BMD } & Age & -0.006 & 0.003 & -0.363 & -2.242 & 0.036 \\
& 25OHD & 0.011 & 0.004 & 0.422 & 2.444 & 0.022 \\
\hline \multirow{2}{*}{ PT BMD } & Age & -0.008 & 0.003 & -0.436 & -2.551 & 0.019 \\
& Gender & -0.179 & 0.075 & -0.474 & -2.385 & 0.027 \\
\hline
\end{tabular}

Abbreviations: DF, distal femur; PT, proximal tibia; BMD, bone mineral density; 25OHD, 25-hydroxyvitamin D.

Table 6 Comparison of BMD Between Complete Lesion and Incomplete Lesion Groups

\begin{tabular}{|l|c|c|c|c|}
\hline Injury Severity & DF BMD $\left(\mathbf{g} / \mathbf{c m}^{2}\right)$ & PT BMD $\left(\mathbf{g} / \mathbf{c m}^{2}\right)$ & Femoral Neck BMD $\left(\mathbf{g} / \mathbf{c m}^{2}\right)$ & Total Hip BMD $\left(\mathbf{g} / \mathbf{c m}^{2}\right)$ \\
\hline Complete $(\mathrm{n}=20)$ & $0.856 \pm 0.144$ & $0.835 \pm 0.141$ & $0.781 \pm 0.122$ & $0.926 \pm 0.151$ \\
Incomplete $(\mathrm{n}=16)$ & $0.759 \pm 0.157$ & $0.757 \pm 0.175$ & $0.709 \pm 0.088$ & $0.877 \pm 0.145$ \\
$\mathrm{t}$ & 1.933 & 1.469 & 1.996 & 0.988 \\
$\mathrm{P}$ & 0.062 & 0.151 & 0.054 & 0.330 \\
\hline
\end{tabular}

Abbreviations: DF, distal femur; PT, proximal tibia; BMD, bone mineral density. 
In the present study, a longitudinal comparison of DF, PT, and hip BMDs in eight patients with SCI showed that the BMDs at the DF and PT were decreased significantly after six months by $11.4 \%$ and $10.8 \%$, respectively, from those at baseline. The BMDs at the femoral neck and total hip had downward trends, but no statistical difference was found. A longitudinal study of 13 patients with acute SCI showed that the decrease in the BMD at the PT was $0.4 \%$ $3.4 \% /$ month, and that the decrease in the BMD at the DF was $0.9 \%-2.8 \% /$ month ${ }^{10}$ which was similar to the results of our present study. A prospective study of 15 patients with SCI within six months found that the BMD at the PT was decreased by $5.3 \%$ from baseline after six weeks. ${ }^{19}$ Another longitudinal study of six patients from 33.5 days to 532 days after SCI found that the BMDs at the DF and PT were reduced by $27 \%$ and $32 \%$, respectively. ${ }^{20}$ The sample sizes of the above studies were relatively small, and there has been no relevant report among Chinese patients. Our present study showed that the bone loss in the DF and PT was more pronounced than that in the hip by longitudinal comparison, which may explain the relatively higher fracture risks in the DF and PT regions. No statistical difference was found in the changes of hip BMD, which may also be related to the small number of cases or the short interval of BMD measurements in our present study. Further research is needed to understand the mechanisms conferring differences in bone-loss patterns in these specific areas, which may help to develop new treatments to delay bone loss after SCI.

The coupling of bone resorption and bone formation is unbalanced in SCI patients. This unbalanced bone remodeling causes continuous bone loss and ultimately leads to osteoporosis, and the most affected areas are the DF and $\mathrm{PT}^{8}{ }^{8}$ The present study showed that the BMD at the DF was negatively correlated with age and PTH and positively correlated with 25OHD, ALP, and PINP; the BMD at the PT was negatively correlated with age and positively correlated with PINP. Further multiple linear regression analysis eliminated potential interactions between these factors. The results showed that age and 25OHD to be the influencing factors of DF BMD and age and gender to be the influencing factors of PT BMD. Vitamin D plays an important role in regulating bone metabolism. Vitamin D deficiency can disrupt the balance between calcium and phosphorus, cause bone mineralization disorders, and increase the risk of falls and fractures. Many previous studies have observed 25OHD deficiency in both acute and chronic SCI patients, with an incidence ranging from
$33 \%$ to $93 \% .{ }^{21-23}$ However, no such study has been reported in a Chinese population. In the present study, the $25 \mathrm{OHD}$ deficiency accounted for $88.89 \%$ of SCI patients, which is significantly higher than the proportion of $25 \mathrm{OHD}$ deficiency (48.1\%) in the healthy population in Beijing, China. ${ }^{24}$ Vitamin D deficiency may be related to race, disease course, insufficient sunshine, and eating habits. In a study of patients with chronic SCI, no correlation was found between serum 25OHD levels and femoral or tibial BMD. ${ }^{25}$ However, the present study found that DF BMD was positively correlated with $25 \mathrm{OHD}$, which may be related to the fact that the population in the present study mostly consisted of patients in the acute phase of SCI. Studies have shown that vitamin-D supplementation in SCI patients can effectively increase serum 25OHD levels. ${ }^{26}$ Combined with the results of our present study, supplementation of vitamin D in early-stage SCI patients may help to prevent further bone loss. Studies have shown that bone mineral loss in female SCI patients is more significant than in male SCI patients. ${ }^{27}$ This study suggested that female patients with SCI should pay special attention to the BMD of PT and carefully protect their knees. Age is an important factor influencing BMD. For older patients with SCI, more attention should be paid to BMD surrounding the knee in daily life and any form of physical training, so as to prevent fractures in this area.

A few previous studies showed there to be no significant difference between complete and incomplete lesions with respect to BMD at the hip, ${ }^{8,28}$ but the effect of injury severity on BMD of DF and PT has only rarely been reported. This study showed there to be no significant difference between the complete lesion and incomplete lesion groups. We consider the most important factor in the stimulation of bone turnover to be the change in bone mechanical stress, which may not be related to the severity of injury. Due to the heterogeneity of different research populations, future research covering a larger sample and more detailed hierarchy will be needed.

To the best of our knowledge, our present study is the first study to report BMDs around the knee of SCI patients in China. An advantage of our present study is that we selected patients during the first year of SCI, which may better reflect the patterns and characteristics of acute-SCI-related bone loss. Our findings of reduced BMDs at high-risk sites such as the DF and PT in earlystage SCI patients suggest the need for targeted early intervention to reduce bone loss. Additionally, our sample population represented characteristics of the vast 
majority of SCI patients, as more than $70 \%$ of SCI patients consist of men, and approximately $50 \%-70 \%$ of SCI cases occur in individuals aged 15-35 years. ${ }^{10}$ One limitation of our present study was that we only assessed a limited sample for our longitudinal observation. Therefore, future studies with larger sample sizes are needed to determine trends and characteristics of BMD changes in SCI patients.

\section{Conclusion}

The rapid loss of BMD at the PT and DF within the first year of SCI occurred significantly earlier than any decrease in hip BMD. Hence, it is necessary to monitor BMDs at the DF and PT in patients with early-stage SCI to assess early bone loss. Treatment should be performed in the early postinjury stage and within the time window before bone loss becomes severe to delay the progression of osteoporosis after SCI and to reduce the risk of future fractures. This strategy should also be combined with the detection of bone biochemical markers to determine the therapeutic effects of rehabilitation training or drug interventions on osteoporosis after SCI.

\section{Data Sharing Statement}

The data used to support the findings of this study are available from the corresponding author (Xin Zheng, Email: zhengxincrrc@126.com) upon request.

\section{Disclosure}

Xin Zheng and Hongjun Zhou should be considered joint corresponding authors. The authors report no conflicts of interest in this work.

\section{References}

1. Comarr AE, Hutchinson RH, Bors E. Extremity fractures of patients with spinal cord injuries. Am J Surg. 1962;103(6):732-739. doi:10.1016/0002-9610(62)90256-8

2. Gifre L, Vidal J, Carrasco J, et al. Incidence of skeletal fractures after traumatic spinal cord injury: a 10-year follow-up study. Clin Rehabil. 2014;28(4):361-369. doi:10.1177/0269215513501905

3. Troy KL, Morse LR. Measurement of bone: diagnosis of SCI-induced osteoporosis and fracture risk prediction. Top Spinal Cord Inj Rehabil. 2015;21(4):267-274. doi:10.1310/sci2104-267

4. Cirnigliaro CM, Myslinski MJ, La Fountaine MF, Kirshblum SC, Forrest GF, Bauman WA. Bone loss at the distal femur and proximal tibia in persons with spinal cord injury: imaging approaches, risk of fracture, and potential treatment options. Osteoporos Int. 2017;28 (3):747-765. doi:10.1007/s00198-016-3798-x

5. Maïmoun L, Couret I, Micallef JP, et al. Use of bone biochemical markers with dual-energy $\mathrm{x}$-ray absorptiometry for early determination of bone loss in persons with spinal cord injury. Metabolism. 2002;51 (8):958-963. doi:10.1053/meta.2002.34013
6. Roberts D, Lee W, Cuneo RC, et al. Longitudinal study of bone turnover after acute spinal cord injury. J Clin Endocrinol Metab. 1998;83(2):415-422. doi:10.1210/jcem.83.2.4581

7. Biering-Sørensen F, Bohr HH, Schaadt OP. Longitudinal study of bone mineral content in the lumbar spine, the forearm and the lower extremities after spinal cord injury. Eur J Clin Invest. 1990;20 (3):330-335. doi:10.1111/j.1365-2362.1990.tb01865.x

8. Dauty M, Perrouin Verbe B, Maugars Y, Dubois C, Mathe JF. Supralesional and sublesional bone mineral density in spinal cord-injured patients. Bone. 2000;27(2):305-309. doi:10.1016/ S8756-3282(00)00326-4

9. Lobos S, Cooke A, Simonett G, Ho C, Boyd SK, Edwards WB. Trabecular bone score at the distal femur and proximal tibia in individuals with spinal cord injury. J Clin Densitometry. 2019;22 (2):249-256. doi:10.1016/j.jocd.2018.04.002

10. Edwards WB, Schnitzer TJ, Troy KL. Bone mineral and stiffness loss at the distal femur and proximal tibia in acute spinal cord injury. Osteoporosis Int. 2014;25(3):1005-1015. doi:10.1007/s00198-0132557-5

11. ASIA and ISCoS International Standards Committee. The 2019 revision of the international standards for neurological classification of spinal cord injury (ISNCSCI)-What's new? Spinal Cord. 2019;57 (10):815-817. doi:10.1038/s41393-019-0350-9

12. Frotzler A, Berger M, Knecht H, Eser P. Bone steady-state is established at reduced bone strength after spinal cord injury: a longitudinal study using peripheral quantitative computed tomography (pQCT). Bone. 2008;43(3):549-555. doi:10.1016/j.bone.2008.05.006

13. Recker R, Lappe J, Davies K, Heaney R. Characterization of perimenopausal bone loss: a prospective study. J Bone Mineral Res. 2000;15(10):1965-1973. doi:10.1359/jbmr.2000.15.10.1965

14. Leblanc AD, Schneider VS, Evans HJ, Engelbretson DA, Krebs JM. Bone mineral loss and recovery after 17 weeks of bed rest. $J$ Bone Mineral Res. 1990;5(8):843-850. doi:10.1002/jbmr.5650050807

15. Vico L, Collet P, Guignandon A, et al. Effects of long-term microgravity exposure on cancellous and cortical weight-bearing bones of cosmonauts. Lancet. 2000;355(9215):1607-1611. doi:10.1016/ S0140-6736(00)02217-0

16. Vestergaard P, Krogh K, Rejnmark L, Mosekilde L. Fracture rates and risk factors for fractures in patients with spinal cord injury. Spinal Cord. 1998;36(11):790-796. doi:10.1038/sj.sc.3100648

17. Morse LR, Battaglino RA, Stolzmann KL, et al. Osteoporotic fractures and hospitalization risk in chronic spinal cord injury. Osteoporos Int. 2009;20(3):385-392. doi:10.1007/s00198-008-0671-6

18. Haider IT, Lobos SM, Simonian N, Schnitzer TJ, Edwards WB. Bone fragility after spinal cord injury: reductions in stiffness and bone mineral at the distal femur and proximal tibia as a function of time. Osteoporosis Int. 2018;29(12):2703-2715. doi:10.1007/s00198-018-4733-0

19. Warden SJ, Bennell KL, Matthews B, Brown DJ, McMeeken JM, Wark JD. Quantitative ultrasound assessment of acute bone loss following spinal cord injury: a longitudinal pilot study. Osteoporosis Int. 2002;13(7):586-592. doi:10.1007/s001980200077

20. Garland DE, Adkins RH, Scott M, Singh H, Massih M, Stewart C. Bone loss at the os calcis compared with bone loss at the knee in individuals with spinal cord injury. J Spinal Cord Med. 2004;27 (3):207-211. doi:10.1080/10790268.2004.11753749

21. Bauman WA, Zhong YG, Schwartz E. Vitamin D deficiency in veterans with chronic spinal cord injury. Metabolism. 1995;44 (12):1612-1616. doi:10.1016/0026-0495(95)90083-7

22. Nemunaitis GA, Mejia M, Nagy JA, Johnson T, Chae J, Roach MJ. A descriptive study on vitamin $\mathrm{D}$ levels in individuals with spinal cord injury in an acute inpatient rehabilitation setting. $j$ Inj Funct Rehabil. 2010;2(3):202-208. doi:10.1016/j.pmrj.2010.01.010

23. Javidan AN, Sabour H, Latifi S, et al. Evaluation of bone mineral loss in patients with chronic traumatic spinal cord injury in Iran. $J$ Spinal Cord Med. 2014;37(6):744-750. doi:10.1179/2045772313Y.0 000000192 
24. XiaoQiu C, Xiaowei D, Jiajing Y, Xia L, Yahan W. A survey on serum $25(\mathrm{OH})$ D3 level of adults. Med J Chin People Armed Police Forces. 2017;28(6):582-586.

25. Zleik N, Weaver F, Harmon RL, et al. Prevention and management of osteoporosis and osteoporotic fractures in persons with a spinal cord injury or disorder: a systematic scoping review. J Spinal Cord Med. 2019;42(6):735-759. doi:10.1080/10790268.2018.1469808

26. Bauman WA, Emmons RR, Cirnigliaro CM, Kirshblum SC, Spungen AM. An effective oral vitamin D replacement therapy in persons with spinal cord injury. J Spinal Cord Med. 2011;34 (5):455-460. doi:10.1179/2045772311Y.0000000032
27. Coupaud S, McLean AN, Purcell M, Fraser MH, Allan DB Decreases in bone mineral density at cortical and trabecular sites in the tibia and femur during the first year of spinal cord injury. Bone. 2015;74:69-75. doi:10.1016/j.bone.2015.01.005

28. Kaya K, Aybay C, Ozel S, Kutay N, Gokkaya O. Evaluation of bone mineral density in patients with spinal cord injury. J Spinal Cord Med. 2006;29(4):396-401. doi:10.1080/10790268.2006.11753888

\section{Publish your work in this journal}

The International Journal of General Medicine is an international, peer-reviewed open-access journal that focuses on general and internal medicine, pathogenesis, epidemiology, diagnosis, monitoring and treatment protocols. The journal is characterized by the rapid reporting of reviews, original research and clinical studies across all disease areas. The manuscript management system is completely online and includes a very quick and fair peer-review system, which is all easy to use. Visit http://www.dovepress.com/ testimonials.php to read real quotes from published authors.

Submit your manuscript here: https://www.dovepress.com/international-journal-of-general-medicine-journal 